\title{
Journal of Imaging: Multidisciplinary Open Access in Image Acquisition, Processing and Understanding
}

\author{
Gonzalo Pajares \\ Department of Software Engineering and Artificial Intelligence, Faculty of Informatics, \\ University Complutense of Madrid, Madrid 28040, Spain; E-Mail: pajares@ucm.es; \\ Tel.: +34-1-394-7546; Fax: +34-1-394-7547
}

Received: 15 February 2015 / Accepted: 27 February 2015 / Published: 5 March 2015

The term "imaging", specifically in relation to the journal, is considered in its broadest sense. Image processing, image understanding and computer vision are all terms related to imaging acquisition, its processing and the extraction of relevant information from the scene to obtain the underlying knowledge. All tasks related to the above items are oriented toward specific applications in a broad range of areas and topics.

The progress made in these areas has been enormous. This has been aided by technological developments of imaging devices, the computational power of machines and the scientific advances and developments in all related fields.

The Journal of Imaging is conceived as an efficient vehicle in the scientific community for the communication and transmission of the progress and research results in the topics covered. In this regard, not only will the scientific community benefit from the existence of this journal, but also industry and other areas, which will obtain the appropriate knowledge and use of the advances described in the journal.

The aim of the multi/disciplinary open access and peer-reviewed Journal of Imaging is to publish reviews, original research papers, communications, case reports, letters, and short notes in all fields of imaging research, striving to incorporate technological innovations and technical methods and approaches covering the following three main topics: acquisition, processing and understanding.

Image acquisition and formation [1,2]: Single/multi frames and video sequences are captured by specific devices and sensors. Here it is worth mentioning, among others: imaging physics, cameras and video cameras (ultraviolet, visible, multispectral, infrared, thermal), sonography devices; biomedical optical devices (microscopy, X-ray, computer tomography or fluoroscopy techniques); sonar and radar imaging, microscopes or scanners (including hyperspectral systems, magnetic resonance and profiling 
sonars). Image generation is obtained by signal sampling or digital devices based on CCD and CMOS technologies where color images or multispectral bands are produced.

Image processing [2-7]: Computational techniques oriented to modify the image content with the aim of its improvement or arrangement for subsequent tasks. It represents the low level of the global computer vision process. The following is a list of image processing topics: enhancement in the spatial and frequency domains, restoration, superresolution, segmentation, color and multispectral transformations, morphological operations, multiresolution (wavelet and pyramidal transform for analysis and synthesis), texture description, feature detection, representation and extraction, real-time, fusion or compression.

Image understanding [7-10]: Represents the high level in the global computer vision process. It is focused on image interpretation and analysis tasks. The following is a list of research topics covered under this area and grouped in subtopics: (a) temporal analysis, including motion, object tracking, change detection; (b) shape-from- $X$ : to obtain 2D and 3D representations, where $X$ means stereovision, shading, focus, texture, photometric stereo, motion, knowledge; (c) pattern and object recognition: statistical and probabilistics approaches, machine learning, structural analysis or bioinspired approaches.

The range of applications is extensive. Applications could include, but are not limited to: robotics; astronomy; agriculture and forestry; forest monitoring; surveillance; measurement; recognition; biometrics; medical and biomedical analysis, behaviours and diagnostic [11]; thermography; photogrammetry [12,13]; cartography; geographical observation; monitoring (disaster, environmental, wildlife); protection and security; quality in manufacturing processes; search and rescue; target tracking; traffic control; data visualization or document processing and analysis. Most applications require specific hardware/software architectures and implementations, sometimes oriented toward real-time processing [14]. These additional topics are also covered by this journal.

Logistics, with or without processing involving images and video sequences, becomes another challenge. Indeed, when images and videos are captured, they must be stored in-situ or transmitted with or without compression for remote processing with subsequent retrieval and decompression. In this regard, storage, coding/decoding, compression, transmission, retrieval and decompression are typical logistics topics.

Under the Open Access format, Journal of Imaging offers an interesting opportunity to scientists, engineers, students, developers, end users and other interested persons. It covers a broad range of topics and applications, which, grouped in a unique journal, facilitate a global overview of its specific multidisciplinary areas. This represents an important added value in the field of imaging.

Special issues are designed present different viewpoints upon a single subject, giving rise to an overview of the subject and its applications.

The Editorial Board consists of a team of experts in imaging and computer vision approaches. Together with the peer-review process, the quality of the published works is guaranteed and authors can achieve a high degree of satisfaction.

Journal of Imaging solicits and encourages authors worldwide to contribute original research manuscripts on the topics described above, offering the opportunity to share the findings and contributions within the community interested in the areas and topics addressed. The success of the journal is expected to result from the anticipated cooperation of everyone involved. 


\section{Conflicts of Interest}

The author declares no conflict of interest.

\section{References}

1. Sinha, P.K. Image Acquisition and Preprocessing for Machine Vision. SPIE Press: Bellingham, WA, USA, 2012.

2. Gonzalez, R.C.; Woods, R.E. Digital Image Processing, 3rd ed.; Prentice Hall: Upper Saddle River, NJ, USA, 2007.

3. IET Image Processing. Available online: http://digital-library.theiet.org/content/journals/iet-ipr (accessed on 16 February 2015).

4. Pratt, W.K. Digital Image Processing, 4th ed.; John Willey and Sons: Hoboken, NJ, USA, 2012.

5. EURASIP Journal on Image and Video Processing. Available online: http://jivp.eurasipjournals.com/ (accessed on 16 February 2015).

6. IEEE Transactions on Image Processing. Available online: http://www.signalprocessingsociety.org/ publications/periodicals/image-processing/ (accessed on 16 February 2015).

7. Pajares, G.; de la Cruz, J.M. Computer Vision: Image Processing and Applications, 2nd ed.; RA-MA: Madrid, Spain, 2007 (in Spanish).

8. IET Computer Vision. Available online: http://digital-library.theiet.org/content/journals/iet-cvi (accessed on 16 February 2015).

9. Computer Vision and Image Understanding. Available online: http://www.journals.elsevier.com/ computer-vision-and-image-understanding/ (accessed on 16 February 2015).

10. Image and Vision Computing. Available online: http://www.journals.elsevier.com/image-andvision-computing/ (accessed on 16 February 2015).

11. IEEE Transactions on Medical Imaging. Available online: http://www.ieee-tmi.org/ (accessed on 16 February 2015).

12. Photogrammetric Engineering \& Remote Sensing. Available online: http://www.asprs.org/ Photogrammetric-Engineering-and-Remote-Sensing/PE-RS-Journals.html (accessed on 16 February 2015).

13. ISPRS Journal of Photogrammetry and Remote Sensing. Available online: http://www.journals.elsevier.com/isprs-journal-of-photogrammetry-and-remote-sensing/ (accessed on 16 February 2015).

14. IEEE Transactions on Circuits and Systems for Video Technology. Available online: http://tcsvt.polito.it/ (accessed on 16 February 2015).

(C) 2015 by the authors; licensee MDPI, Basel, Switzerland. This article is an open access article distributed under the terms and conditions of the Creative Commons Attribution license (http://creativecommons.org/licenses/by/4.0/). 